Document downloaded from:

http://hdl.handle.net/10251/83430

This paper must be cited as:

Triana-Infante, CA.; Pastor Abellán, D.; Varón, M. (2016). Overlap-proof Fiber Bragg Grating Sensing System using Spectral Encoding. IEEE Photonics Technology Letters. 28(7):744747. doi:10.1109/LPT.2015.2508743.

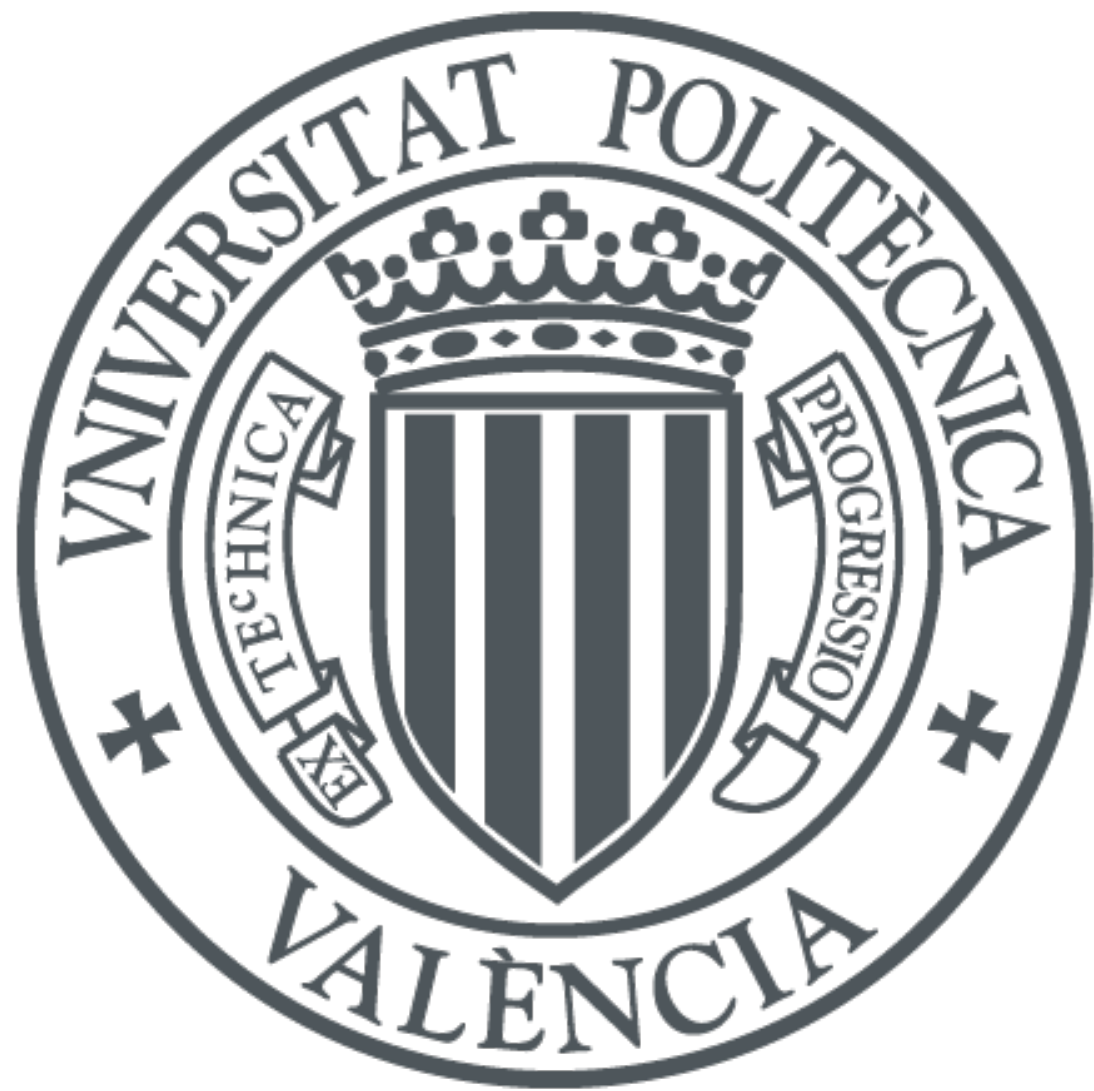

The final publication is available at

http://dx.doi.org/10.1109/LPT.2015.2508743

Copyright Institute of Electrical and Electronics Engineers (IEEE)

Additional Information

(c) 2015 IEEE. Personal use of this material is permitted. Permission from IEEE must be obtained for all other users, including reprinting/ republishing this material for advertising or promotional purposes, creating new collective works for resale or redistribution to servers or lists, or reuse of any copyrighted components of this work in other works. 


\title{
Overlap-proof Fiber Bragg Grating Sensing System using Spectral Encoding
}

\author{
Andrés Triana, Daniel Pastor, Member, IEEE, and Margarita Varón, Member, IEEE
}

\begin{abstract}
In this letter, a spectral encoding of Fiber Bragg Grating (FBG) sensors is proposed in order to solve their wavelength under overlapping conditions. Our technique uses Optical Orthogonal Codes (OOC) to define the spectral shape of the FBG sensors. In this way, we design encoded FBG sensors composed of more than one reflection band; the spectral location of the reflection bands are determined by the OOC, providing each encoded FBG sensor with a unique spectral signature. The demodulation technique for the proposed sensors makes use of their orthogonal nature: it obtains the wavelength position for each sensor by obtaining the auto-correlation product between the reflection spectrum and each sensor's spectral code. These FBG sensors have been manufactured and experimentally validated. The results demonstrate the overlap-proof nature of the encoded sensors. Furthermore, the very simple demodulation technique provides highly accurate wavelength detection and can be implemented in real-time detection systems.
\end{abstract}

Index Terms-Fiber Bragg grating (FBG), optical fiber sensor, overlap-proof, spectral encoding, demodulation.

\section{INTRODUCTION}

O PTICAL sensing systems use multiplexing schemes, such as Wavelength Division Multiplexing (WDM) and Time Division Multiplexing (TDM), in order to increase the number of available sensors, reducing the overall cost of the system. WDM and TDM techniques are fully compatible and have been employed successfully in the widely accepted FBG sensing networks [1]. Indeed, as the measurement principle of FBG sensors is related to the shift in their reflected wavelength, they are intrinsically suitable for WDM multiplexing [2], where each sensor is constrained to a spectral operational range and the total incident spectrum is divided into the working range assigned to each sensor.

Despite the wide use of WDM and TDM techniques in optical fiber sensing, an important drawback is related to the spectral range distribution for each sensor. When the total spectrum is divided, what is usually employed is the maximum working range for all the sensors plus a guard band in between in order to avoid overlapping between two reflected wavelengths. This results in an oversized spectral range for each sensor, which leads to an inefficient use of the total spectrum. TDM techniques require more complexity in the detection system due to the need of synchronization between the emitted pulsed source and the interrogator.

Andrés Triana and Daniel Pastor are with the Optical and Quantum Communications Group (iTEAM Research Institute), Universidad Politécnica de Valencia, Valencia 46022, Spain (e-mail: catrianai@unal.edu.co)

Andrés Triana and Margarita Varón are with the CMUN Research Group, Universidad Nacional de Colombia. Cra 30 No. 45-06, 111321 Bogotá DC, Colombia

Manuscript received;

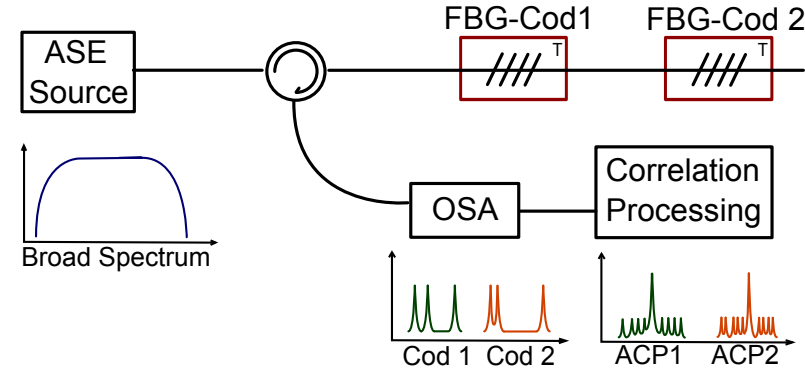

Figure 1. Schematic diagram of the setup used to measure the encoded FBG sensors. The reflection spectra of the encoded sensors is measured and its central wavelength is obtained from the auto-correlation product.

On the other hand, there is another multiplexing technology in optical communications, fully compatible with WDM and TDM, namely optical Code Division Multiple-Access (CDM) [3]. In optical CDM systems, a codeword is given to each user in the system instead of a wavelength range or a time slot. In this way, the channel can be used by all the users at the same time, each one sending information with a distinct encoding. There are some important advantages to including Code Division Multiplexing in the design of an FBG sensor. Encoding the FBG sensors' spectra could provide them with a unique signature that makes their identification easier in the sensing network. Another advantage comes from the fact that the encoding sensor is not as simple as a single FBG, therefore it contains more information and the measurements will gain robustness regarding interference or distortion in the spectrum that could affect the accuracy of the system. Finally, overlap between sensors is possible without losing their reference and without using sophisticated identification algorithms [4], [5]. This is definitely attractive in the design of FBG sensing networks because it allows a more efficient use of the spectrum and consequently the number of sensors deployed in the same available spectrum can be increased.

Basically, each encoded FBG sensor is a grating device composed of several reflection bands distributed according to a given Optical Orthogonal Code (OOC). The set of gratings are written at the same location of the optical fiber so that environmental conditions affect all the reflection bands equally. In this letter, spectrally encoded FBG sensors based on OOC [6] are proposed, manufactured and experimentally validated according to the setup shown in Fig. 1. It is also shown that the auto-correlation product between each sensor codeword and the total reflection spectrum demodulates the central wavelength of each sensor. Finally, experimental results proving the overlap-proof behavior of the proposed sensors are presented and discussed. 

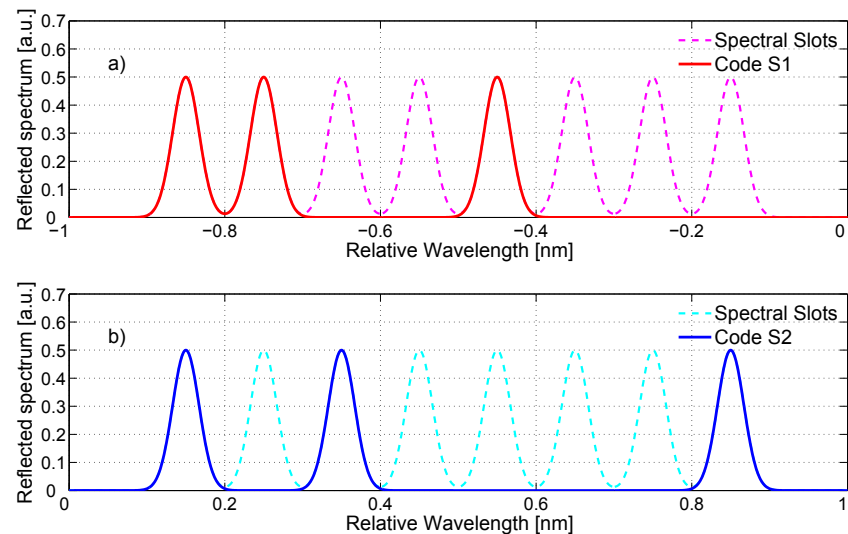

Figure 2. Spectral shape of the FBG encoded sensors, reflection subbands correspond to the binary encoding of $S 1=$ [11001000] and $S 2=$ [10100001]. Each plot is represented regarding a central (zero) wavelength, Sensor $S 1$ (a) is located at a distance of $-0.5 \mathrm{~nm}$ and sensor $S 2$ (b) at a distance of $0.5 \mathrm{~nm}$ from the zero wavelength

\section{ENCODED FBG SENSORS}

The basic intention behind FBG sensor encoding is to provide each sensor with a unique spectral shape, totally discernible from that of its neighboring sensors in the spectrum. Optical Orthogonal Codes (OOC) are used in CDM communications to spread a signal in the time domain with a certain pattern [7]. In our case OOCs are used to design the spectral conformation of FBG sensors.

In the simplest case, each pair of encoded sensors adjacent in the spectrum will allow the total identification of their central wavelength even in overlapped scenarios thanks to their orthogonal behavior. Thus, the number of sensors in the available spectrum could be doubled. Obtaining the orthogonal codewords is performed using the combinatorial method [8]. For instance, in this research, the codewords $S 1=$ [11001000] and $S 2=$ [10100001] were used to define the shape of the two encoded FBG sensors depicted in Fig. 2, where each sensor features 8 equidistant spectral slots with reflection sub-bands corresponding to the $1 \mathrm{~s}$ in the codewords $S 1$ and $S 2$.

The total reflection spectrum $R(\lambda)$ is the sum of all the sub-bands belonging to the sensors in the spectrum, and could be written in general, for $K$ sensors, as

$$
\begin{aligned}
& R(\lambda)= \\
& \sum_{j=1}^{K} \sum_{i=-N / 2+1}^{N / 2} c_{j}(i) \cdot R^{\prime} \cdot g\left(\lambda-\lambda_{B j}-\Delta \lambda_{B j}-(\delta \lambda \cdot i-\delta \lambda / 2)\right)
\end{aligned}
$$

where $g(\lambda)$ is the normalized spectral shape of the sub-bands of the sensors, $R^{\prime}$ is the peak reflectivity, $\lambda_{B j}$ is the Bragg wavelength, $\delta \lambda$ is the spectral separation between sub-bands, and $c_{j}(i)$ is the binary code value corresponding to the $j^{\text {th }}$ encoded sensor and $i^{\text {th }}$ sub-band of the code (note that the sum for the $N$ sub-bands goes from $-N / 2+1$ to $N / 2$ in order to take into account that the number of sub-bands may be even or odd).

The demodulation technique for the encoded FBG sensors is related to their orthogonal nature. These codes satisfy the

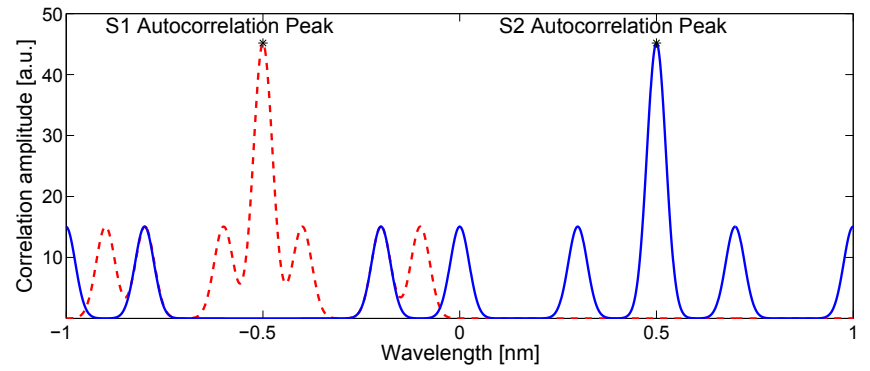

Figure 3. Auto-correlation product for the codewords S1 (dashed red) and S2 (solid blue) when their central wavelengths are separated by $1 \mathrm{~nm} .( \pm 0.5$ $\mathrm{nm}$ from the zero wavelength.)

auto- and cross-correlation properties, which means that the product of the codeword with itself gives as a result a unique peak (namely the auto-correlation peak ACP). The product between two different codewords (or even between shifted versions of the same codeword) is constrained to a lower value, which is the cross-correlation product (XC) [7]. From this concept, we can identify the central position of the encoded sensors by evaluating the auto-correlation product between each codeword and the total measured reflection spectrum (similar to the approaches presented in [9], [10]). Fig. 3 shows the ACP and XC wings for sensors $S 1$ and $S 2$ located at a spectral distance of $1 \mathrm{~nm}$.

The Identification Function $(I F)$ (2) performs the autocorrelation product for each sensor.

$$
\begin{aligned}
& I F_{j}\left(\lambda^{\prime}\right)= \\
& \int\left(\sum_{i=-N / 2+1}^{N / 2} c_{j}(i) \cdot g\left(\lambda-\lambda^{\prime}-\delta \lambda \cdot(i-1 / 2)\right)\right) \cdot R(\lambda) d \lambda
\end{aligned}
$$

This calculation was performed in our system (Fig. 1) for each sensor $j$. The maximum of $I F_{j}\left(\lambda^{\prime}\right)$, i.e. the ACP value, matches $\lambda_{B j}+\Delta \lambda_{B j}$, which is the central position of each sensor. The $I F$ calculation is performed by the scalar product between the readout of the reflected spectrum measured by the OSA and each one of the $\mathrm{K}$ encoded sensor profiles pre-charged in a computer, so it does not represent any computational complexity.

The maximum number of overlapped sensors with proper orthogonality (XC/ACP ratio) depends in general on the number of sub-bands and spectral slots $(\mathrm{N})$ according with the OOC design. Increasing the number of spectral slots leads to a broader spectrum for each sensor, but it can be compensated reducing the slot separation $\delta \lambda$.

\section{EXPERIMENTAL SETUP AND RESUlts}

\section{A. Manufacturing of encoded FBG sensors}

In order to build the proposed encoded FBG sensors, we write several reflection sub-bands at the same location of the optical fiber. Such devices are known as Super-Imposed FBGs, and have been demonstrated for OCDMA communications systems in [11], [12]. The wavelength shift due to environmental perturbations will be the same for all the sub-bands written 
TABLE I

COMPARISON OF DESIGNEd AND MANUfactured PARAMETERS OF THE FBG ENCODED SENSORS

\begin{tabular}{ccccccc}
\hline & $\begin{array}{c}\text { Medium } \\
\text { Reflectivity } \%\end{array}$ & \multicolumn{2}{c}{$\begin{array}{c}\text { Sub-bands' } \\
\text { FWHM }[\mathrm{pm}]\end{array}$} & \multicolumn{2}{c}{$\begin{array}{c}\text { Sub-bands' } \\
\text { Distance [pm] }\end{array}$} \\
\hline Design S1 & 30 & 40 & 40 & 40 & 100 & 300 \\
FBG S1 & 31.5 & 40.8 & 42.8 & 37.76 & 104 & 294 \\
Design S2 & 30 & 40 & 40 & 40 & 200 & 500 \\
FBG S2 & 30.7 & 40.3 & 39.8 & 39.3 & 194 & 490 \\
\hline
\end{tabular}
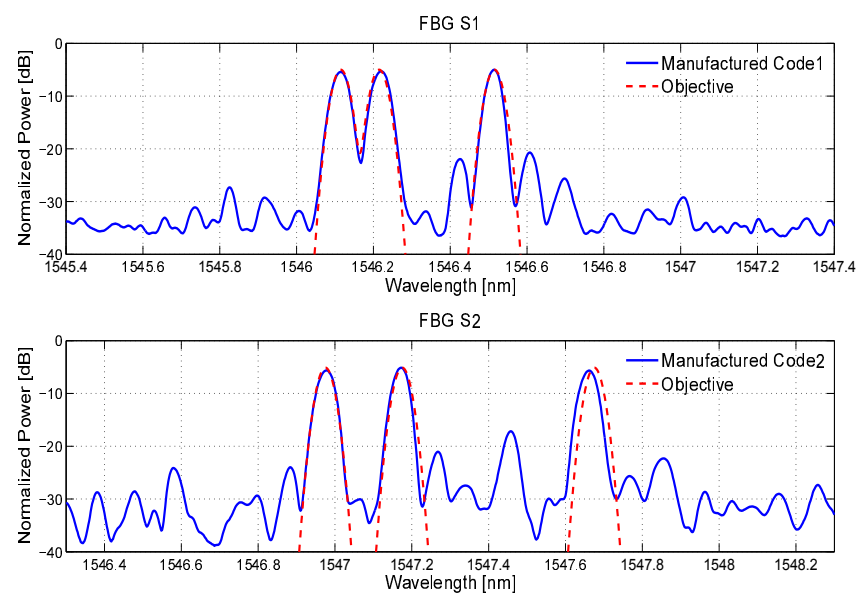

Figure 4. Reflection spectra for the manufactured encoded FBG sensors $S 1$ and $S 2$, dashed red lines represent the design objective in both cases.

in the same location. The proposed encoded FBG sensors were designed employing the Discrete Layer Peeling synthesis method (DLP) [13], using the spectra depicted in Fig. 2 as the target response (i.e. Reflectivity $=25 \%$ and $F W H M=40$ $\mathrm{pm})$. The gratings were written on a photosensitive optical fiber by exposure to an Ultra-Violet (UV) laser beam through a phase mask (PM) of $1070 \mathrm{~nm}$. The UV beam was focused before the PM up to $\sim 40 \mu \mathrm{m}$. The amplitude and phase control of each sample was achieved by a double UV exposure of $500 \mathrm{~ms}$ over the same $z$-position, only changing the relative phase of the Bragg period by the proper PM-Optical fiber displacement. In this way, the average UV flux is constant and the average refractive index value remains unaltered [14]. The manufactured gratings are $42 \mathrm{~mm}$ long with central wavelengths at $S 1=1546.5 \mathrm{~nm}$ and $S 2=1547.3 \mathrm{~nm}$ at room temperature. The comparison between the designed and the manufactured sensors is summarized in Table I.

The reflection spectra of the manufactured sensors $S 1$ and $S 2$ is depicted in Fig. 4, together with the design objective used as the input to the DLP synthesis method. Very good agreement was achieved between the objective and the manufactured FBGs. Side lobe suppression levels are greater than $15 \mathrm{~dB}$ for sensor 1 and $12 \mathrm{~dB}$ for sensor 2 due to fabrication imperfections, which means that side lobes in Fig 4. have very low impact on the ACP and XC detection.

\section{B. Experimental Setup}

The experimental setup is represented in Fig. 1. Two enconded FBG sensors are disposed in a serial configuration.
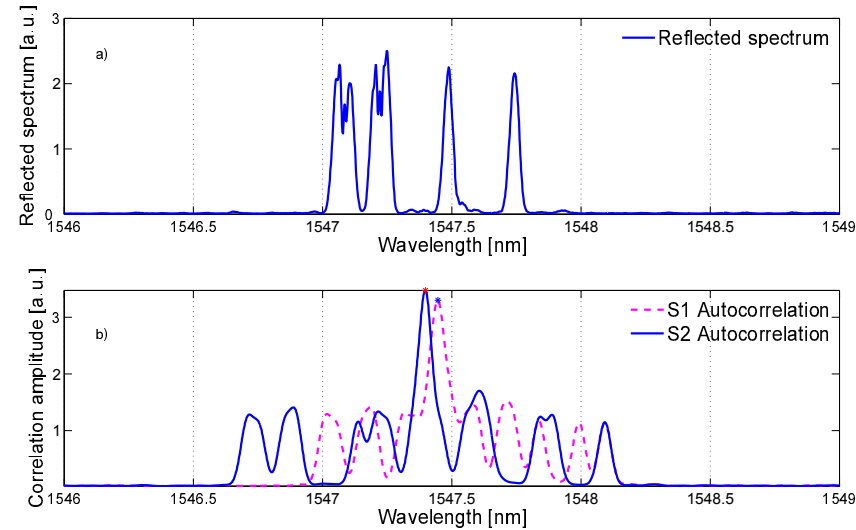

Figure 5. In a), a readout of the two encoded FBG sensors under overlapping conditions; b) the auto-correlation product for this single readout. The ACP for each sensor indicates its central position in the spectrum.
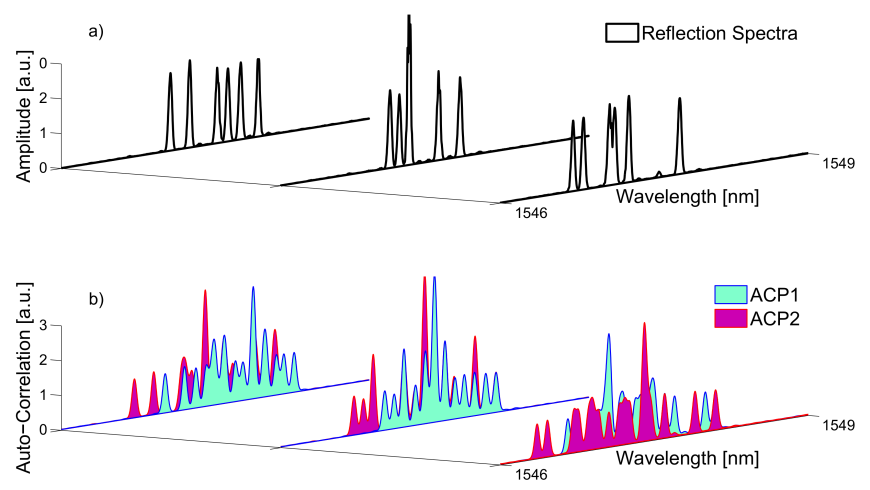

Figure 6. A three step evolution of the overlapping readout between sensors $S 1$ and $S 2$ is sketched in a). In b) there are depicted the auto-correlation products for each encoded FBG sensor regarding each measurement in a).

A C-band Amplified Spontaneous Emission (ASE) source illuminates the two encoded FBG sensors through a circulator used to recover the reflected signal. An Optical Spectrum Analyzer (OSA) ANDO AQ6317C, with $10 \mathrm{pm}$ resolution, acquires spectral readouts of 2000 points within a $3 \mathrm{~nm}$ span, which means a wavelength sampling of $1.5 \mathrm{pm}$. The OSA is connected to a computer where the auto-correlation algorithm is performed for each readout so that the ACP for each codeword is computed.

The encoded FBG sensors were positioned inside thermal chambers to stabilize their temperature and attached to micrometric translation stages. The end connections in the setup are covered with index-matching oil to prevent back reflections. During the test the center wavelengths were shifted around $1547.5 \mathrm{~nm}$ to set them close to each other in such a way that stretching the sensor $S 1$, their wavelength cross over the central wavelength of sensor $S 2$, and vice-versa. Fig. 5 a) shows a single readout of the spectrum while the two sensors are overlapping. In b) are plotted their auto-correlation products, indicating the central wavelength for each sensor.

\section{Results}

With the configuration described in Section III-B, the sensors were placed in adjacent positions in the spectrum. One 

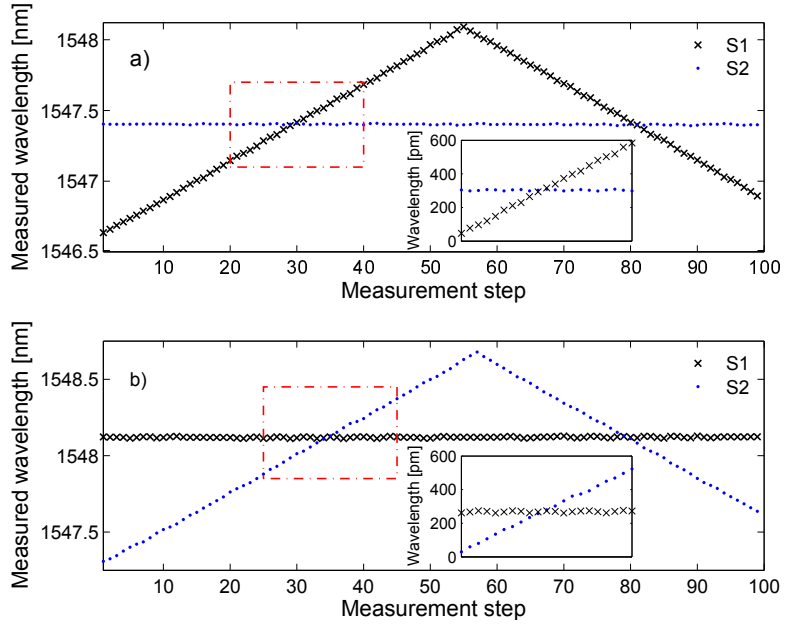

Figure 7. Measured wavelength for the two sensors in overlapping conditions. In a), a swept of sensor $S 1$ while sensor $S 2$ is fixed is depicted. In b) the analog scenario is plotted, sensor $S 2$ cross back and forth $S 1$ 's wavelength.

of them is fixed and the second one follows a linear trajectory crossing completely the wavelength of the fixed sensor. Fig. 6 shows the process of overlapping of the two sensors: sensor $S 1$ is shifted to the right, crossing the wavelength of sensor $S 2$. Fig. 6 a) depicts the reflection spectrum readout and Fig. $6 \mathrm{~b}$ ) plots the auto-correlation identification function for each sensor for three different measurements over the wavelength swept.

The complete set of measurements is presented in Fig. 7 a): the sensor $S 2$ is fixed at $1547.4 \mathrm{~nm}$ and sensor $S 1$ follows a linear path from $1546.63 \mathrm{~nm}$ to $1548.09 \mathrm{~nm}$ and back to $1546.89 \mathrm{~nm}$. Both sensors are temperature controlled during the tests. The strain shift was applied to the moving sensor in steps of $\sim 20 \mu \epsilon$. The same holds for the analogous experiment, depicted in Fig. 7 b), where sensor $S 2$ goes from $1547.3 \mathrm{~nm}$ to $1548.67 \mathrm{~nm}$ and back to $1547.63 \mathrm{~nm}$ with $S 1$ fixed at $1548.12 \mathrm{~nm}$. The insets in Fig. 7 detail the measured wavelength in the overlapping region.

These tests confirm the excellent behavior of the encoded FBG sensors in overlapping scenarios. Because the codes used in their design are completely orthogonal, the identification algorithm obtains the central position for each one even if their sub-bands are partially or fully overlapping.

In order to derive the error of the detection process we compared the obtained results for the overlapping scenario against the error obtained for individual measurements of the same sensors (parallel configuration was utilized to access individual and total reflection from the 2 sensors simultaneously), obtaining a standard deviation difference between the two cases of $4.40 \mathrm{pm}$. In this value, the dominant source of error is the $\mathrm{XC}$ interference over the ACP as discussed, however, other potential sources of error are the reflectivity reduction of the sub-bands when they are overlapped in a serial arrangement of sensors and the wavelength and amplitude accuracy of the manufactured devices. The only difference when using parallel configuration is the use of passive splitting components (a 50:50 coupler in this case). In this way, the encoded FBG sensors will work the same way in the spectrum but the reflectivity can be maximized and the signal to noise ratio consequently enlarged.

\section{CONCLUSION}

We have developed an approach to designing encoded FBG sensors which have the property of being orthogonal, so that their wavelengths can be detected under overlapping conditions. A very fast and simple identification algorithm for the proposed sensors was described, whose operation is based on the auto-correlation product. Therefore it can be implemented easily in any embedded system. The proposed sensors were manufactured as super-imposed FBGs, their spectral features were measured, and their behavior in overlapping conditions was verified. The maximum error in detection was $4.4 \mathrm{pm}$ compared to individual measurement of FBG sensors with the same spectral characteristics.

The spectral overlapping achieved with the proposed encoded sensors allows increasing the number of sensors deployed in an optical sensing network, since the total spectrum can be used more efficiently.

\section{ACKNOWLEDGMENTS}

The authors wish to thank the Spanish MCINN for support through projects TEC2013-42332-P and TEC 2009-12169.

\section{REFERENCES}

[1] A.Kersey et al., "Fiber grating sensors," Lightwave Technology, Journal of, vol. 15, no. 8, pp. 1442-1463, Aug 1997.

[2] T.Erdogan, "Fiber grating spectra," Lightwave Technology, Journal of, vol. 15, no. 8, pp. 1277-1294, Aug 1997.

[3] L.Chen, "Optical Code-Division Multiple-Access Enabled by Fiber Bragg Grating Technology," in Optical Code Division Multiple Access Fundamentals and Applications. CRC Press, Dec. 2005, pp. 111-164-.

[4] D.Liu et al., "A Fiber Bragg Grating Sensor Network Using an Improved Differential Evolution Algorithm," Photonics Technology Letters, IEEE, vol. 23, no. 19, pp. 1385-1387, Oct 2011.

[5] H.Jiang et al., "Wavelength detection in spectrally overlapped FBG sensor network using Extreme Learning Machine," Photonics Technology Letters, IEEE, vol. 26, no. 20, pp. 2031-2034, Oct 2014.

[6] M.Best et al., "Bounds for binary codes of length less than 25," Information Theory, IEEE Transactions on, vol. 24, no. 1, pp. 81-93, Jan 1978.

[7] W.Kwong and G.-C.Yang, "Optical CDMA Codes," in Optical Code Division Multiple Access Fundamentals and Applications, P. R.Prucnal, Ed. CRC Press, Dec. 2005, pp. 55-80-.

[8] F.Chung et al., "Optical orthogonal codes: design, analysis and applications," Information Theory, IEEE Transactions on, vol. 35, no. 3, pp. 595-604, May 1989.

[9] C.Huang et al., "Demodulation of fiber Bragg grating Sensor using Cross-Correlation Algorithm," Photonics Technology Letters, IEEE, vol. 19, no. 9, pp. 707-709, May 2007.

[10] R.Cheng et al., "Interrogation of Ultrashort Bragg Grating Sensors Using Shifted Optical Gaussian Filters," Photonics Technology Letters, IEEE, vol. 27, no. 17, pp. 1833-1836, Sept 2015.

[11] D.Meghavoryan and A.Daryan, "Superimposed fiber Bragg grating simulation by the method of single expression for optical CDMA systems," Photonics Technology Letters, IEEE, vol. 15, no. 11, pp. 1546-1548, Nov 2003.

[12] S.Ayotte et al., "Experimental verification and capacity prediction of FEOCDMA using superimposed FBG," Lightwave Technology, Journal of, vol. 23, no. 2, pp. 724-731, Feb 2005.

[13] J.Skaar et al., "On the synthesis of fiber Bragg gratings by layer peeling," Quantum Electronics, IEEE Journal of, vol. 37, no. 2, pp. 165-173, Feb 2001.

[14] R.Baños et al., "Rectangular global envelope super structured FBGs for multiband coherent OCDMA," Photonics Technology Letters, IEEE, vol. 25, no. 5, pp. 512-514, March 2013. 